\title{
Psicoterapia orientada al conflicto - Psicoterapia orientada a la estructura
}

\section{Conflict-oriented psychotherapy - Structure- oriented psychotherapy}

\author{
Gustavo Lanza Castelli \\ Asociación Internacional para el Estudio y Desarrollo de la Mentalización, España
}

\begin{abstract}
Resumen: El presente trabajo propone una diferenciación entre las patologías determinadas por el conflicto y aquellas que se deben a perturbaciones en el nivel de la estructura. En su desarrollo caracteriza una concepción funcional de la estructura y enumera las capacidades que la constituyen. Establece las diferencias y articulaciones entre el conflicto y la estructura, así como entre las perturbaciones que derivan de ellos. Diferencia las estrategias terapéuticas que se aplican en un caso y en otro en base a los siguientes parámetros: encuadre, posición terapéutica, jerarquización de focos, trabajo con los patrones relacionales, tipos de intervenciones. En la parte final se ilustra el abordaje estructural a partir de un caso clínico y se pone el acento en la necesidad de llevar a cabo una estimación precisa del peso de ambos factores en la evaluación inicial, previa al comienzo de la psicoterapia.

Palabras clave: Conflicto, estructura, psicoterapia y conflicto, psicoterapia y estructura.
\end{abstract}

\begin{abstract}
The present work proposes a differentiation between the pathologies determined by the conflict and those that are due to disturbances at the level of the structure. It characterizes a functional conception of the structure and lists the capacities that constitute it. It establishes the differences and articulations between the conflict and the structure, as well as between the disturbances that derive from them. It differentiates the psychotherapeutic strategies that are applied in one case and in another based on the following parameters: framing, therapeutic position, focus hierarchy, work with relational patterns, types of interventions. In the final part, the structural approach is illustrated with a clinical case and the accent is placed on the need to carry out an accurate assessment of the weight of both factors in the initial evaluation, prior to the start of psychotherapy.

Keywords: Conflict, structure, psychotherapy and conflict, psychotherapy and structure.
\end{abstract}

Gustavo Lanza es Psicoanalista. Presidente en la Asociación Internacional para el Estudio y Desarrollo de la Mentalización.

La correspondencia sobre este artículo debe enviarse al autor al e-mail: gustavo.lanza.castelli@gmail.com

(cc) EY-NC-ND Este es un artículo Open Access bajo la licencia CC BY-NC-ND. 
En el psicoanálisis contemporáneo las consideraciones acerca de la estructura psíquica y el funcionamiento mental se han convertido en esenciales para la comprensión de la problemática de los pacientes que nos consultan hoy en día (Horowitz, Kernberg, Weinshel, 1993), junto a las reflexiones referidas a los conflictos intrapsíquicos y los patrones vinculares prototípicos (Grande, 2007; Grupo de trabajo OPD-2, 2008).

Este interés se deja ver en una serie de autores, de orientaciones en parte distintas, dentro del campo psicoanalítico. Entre ellos cabe citar a Fürstenau (1977), Kernberg (1979), Kohut (1989), Mentzos (2017), Pouget-Schors (2002), Rapaport (1967), Resch (2002) y Rudolf (2002a).

Estos y otros autores coinciden en atribuir decisiva importancia al concepto de estructura psíquica - entendida de un modo un tanto diferente en cada uno de ellos - para alcanzar una comprensión psicodinámica amplia del funcionamiento personal e interpersonal, tanto normal como patológico. En ambos casos, resulta esencial articularlo con la noción de conflicto psíquico (Dreher, 2006; Schüßler, 2004), ya que lo que se postula es, en suma, que los diversos trastornos mentales han de ser comprendidos en base a dos mecanismos diferentes: los conflictos intrapsíquicos y las fallas o déficits en la dimensión estructural del psiquismo (Killingmo,1989; Sugarman, 2006).

Si bien los autores mencionados han contribuido al deslinde conceptual de dos tipos de procesos diferentes y de desenlaces patológicos diversos, es importante consignar que en los hechos clínicos concretos nos encontramos con tres clases distintas de perturbaciones: aquellas en que lo decisivo es el conflicto psíquico, con un funcionamiento estructural adecuado; aquellas otras en las que lo fundamental en el padecimiento del paciente lo constituyen las diversas perturbaciones en su estructura y, por último, aquellas en las que la dimensión del conflicto se ve complicada por déficits a nivel estructural, configurando un cuadro que podríamos llamar mixto. Esta última alternativa parece ser la más frecuente en la práctica clínica (Dreher, 2006; Rudolf, 2004).

En lo que sigue, llevo a cabo una caracterización de la estructura psíquica, basándome en los desarrollos de Rudolf (2004, 2013) y del Grupo de trabajo del OPD-2 (2008). En algunos sectores de dicha caracterización realizo algunas articulaciones con los desarrollos de Fonagy y colaboradores (Allen, Fonagy, Bateman, 2008; Bateman, Fonagy, 2006; Fonagy, Gergely, Jurist y Target, 2002), ya que ambos modelos tienen una serie de elementos en común, así como otros en los que cabe hallar una interesante complementariedad (Lanza Castelli, 2016b). Posteriormente establezco una breve comparación entre las patologías en que predomina el conflicto y aquellas en las que lo decisivo es el déficit estructural, tras lo cual llevo a cabo algunas consideraciones sobre la psicoterapia orientada a la estructura, comparándola con la psicoterapia orientada al conflicto. Por último, ilustro estas ideas con un material clínico y realizo algunas consideraciones sobre la importancia de llevar a cabo una evaluación diferencial precisa, así como sobre las diversas estrategias y técnicas que se ponen en juego según los resultados de dicha evaluación.

\section{El concepto de estructura}

Resulta de utilidad postular una concepción funcional de la estructura, aclarando que la misma debe diferenciarse de los contenidos (representaciones, fantasías, afectos, pulsiones, etc.), ya que se refiere al nivel de organización de las funciones psíquicas, que regulan el sentimiento de sí y el comportamiento relacional (Rudolf, 2004, 2013).

Todos los elementos de la estructura pueden caracterizarse como capacidades. Cuando tales elementos estructurales se desarrollan hasta un nivel adecuado y se encuentran integrados, proveen a la personalidad de un conjunto de capacidades psíquicas, necesarias para su regulación y desenvolvimiento (por ej. la capacidad de diferenciar los propios afectos, de empatizar con los demás, de regular el sentimiento de sí, etc.). Entendido en este sentido, el concepto de estructura no debe ser considerado como de índole especulativa o metapsicológica, ya que todas estas capacidades pueden operacionalizarse e indagarse empíricamente (Rudolf, 2002a).

Por lo demás, dichas capacidades pueden diferenciarse en cuatro categorías, cada una de las cuales se subdivide, a su vez, en aquellas relacionadas con el self y las relacionadas con el objeto (Grupo de trabajo del OPD-2, 2008; Rudolf, 2004, 2013): 


\section{Capacidades de aprehensión y pensamiento}

\section{En relación al self:}

a) Capacidad para formar una imagen realista de uno mismo, para aprehender los propios estados mentales y reflexionar sobre ellos (Fonagy et al. 2002; Juen, 2005; Rudolf, 2004).

b) Capacidad para diferenciar y denominar los propios afectos, lo cual alude a la posibilidad de identificar, diferenciar y traducir verbalmente (incluyéndolos en una narrativa) afectos cualitativamente diferenciados entre sí, sea que surjan en el vivenciar de modo aislado o, lo que es habitual, que formen parte de un conglomerado emocional (Fonagy et al., 2002; Grupo de trabajo del OPD-2, 2008; Rudolf, 2004).

c) Capacidad para tener una imagen de la propia identidad y desarrollarla.

\section{En relación al objeto:}

d) Capacidad para diferenciar el self del objeto, para diferenciar pensamientos, sentimientos e impulsos propios de los de los demás.

e) Capacidad para aprehender de modo realista a los demás, diferenciando e integrando sus distintos aspectos (buenos y malos, entre otros).

f) Capacidad para inferir, de modo plausible, los distintos estados mentales responsables de su conducta, de un modo descentrado y no egocéntrico.

g) En ambos casos (relación al self, relación al objeto) es fundamental que los estados mentales sean vivenciados como tales, esto es, como una realidad psíquica, diferenciable de la contundencia de los hechos, por lo cual se torna posible dudar acerca de dichos estados, que adquieren entonces un carácter conjetural. Esto significa que los estados mentales han de ser comprendidos - de modo explícito o implícito- como una construcción, y no como una réplica exacta de los hechos (Allen, Fonagy, Bateman, 2008; Fonagy et al., 2002). Cuando ocurre esto último y las propias representaciones (de sí mismo y de los demás) alcanzan un alto grado de certidumbre subjetiva, que no deja lugar para la duda o la conjetura, Fonagy y colaboradores hablan de un funcionamiento en equivalencia psíquica (Fonagy et al., 2002), que posee la mayor importancia en diversos desenlaces patológicos.

\section{Capacidades de regulación}

\section{En relación al self:}

a) Regulación de los impulsos: capacidad para contener, regular e integrar los propios impulsos, al servicio del self y de la relación con los demás, sea conteniéndolos, sea dándoles curso en la situación adecuada, teniendo en cuenta los propios valores.

b) Regulación de los afectos: implica un grado aceptable de tolerancia a los afectos, la modificación eventual de su duración e intensidad, así como una expresión adecuada de los mismos.

c) Regulación del sentimiento de estima de sí: supone la capacidad de mantener en un nivel satisfactorio el sentimiento de estima de sí y de recuperarlo cuando ha sido mermado por circunstancias internas o externas.

En los tres casos es importante que exista la capacidad de tomar distancia de dichos contenidos (impulsos y afectos), a los efectos de identificarlos mejor, reflexionar sobre ellos, desidentificarse de los mismos, etc.

Del buen funcionamiento de estas capacidades deriva un sentimiento de autoeficacia, de confianza en sí mismo y de autoafirmación, así como la capacidad de responsabilizarse como artífice de las propias acciones (Grupo de trabajo del OPD-2, 2008).

\section{En relación al objeto:}

Se refiere al intercambio emocional con el objeto.

d) Protección de la relación: implica poder proteger la relación de los propios impulsos y afectos, de modo tal que la satisfacción de los primeros y la expresión de los segundos tenga lugar de un modo interpersonalmente satisfactorio. 
e) Resguardo de los intereses, propios y ajenos: supone la posibilidad de mantener y afirmar los propios intereses (deseos, demandas, etc.) sin que se vean postergados en el seno de la relación con el otro. A la vez, implica tener en cuenta los intereses del otro y la satisfacción que desea de los mismos, en un balance adecuado y mutuamente satisfactorio.

f) Capacidad de anticipación: la capacidad para anticipar cómo una exteriorización desiderativa propia o la expresión de un afecto incidirá en el otro, cómo este ha de responder en consecuencia, es esencial para poder regular las propias acciones en el interior de la relación interpersonal.

\section{Capacidades de comunicación emocional}

\section{En relación al self:}

a) Capacidad de generar y vivenciar afectos propios.

b) Capacidad para mantener diálogos internos, en conexión con la propia experiencia emocional y la capacidad para utilizar las propias fantasías como mediadoras entre los estados emocionales y los diseños de acción relacionados con ellos.

c) Capacidad de percibir el propio cuerpo vivido, de vitalizar emocionalmente el self corporal.

\section{En relación al objeto:}

Se refiere al intercambio emocional con el objeto.

d) Capacidad de establecer contacto emocional con el otro, expresando los propios afectos a la vez que uno se abre a ser afectado por los afectos ajenos.

e) Capacidad para expresar los propios afectos y dejarse conmover por los afectos ajenos

f) Capacidad de empatía, esto es, de ingresar emocionalmente en el mundo vivencial del otro, aprehendiendo su propio punto de vista.

\section{Capacidades de vínculo}

\section{En relación al self:}

a) Capacidad para internalizar objetos buenos y para construir y mantener afectos positivos referidos a objetos.

b) Capacidad de establecer lazos internos cualitativamente diferenciados con los objetos internos.

c) Capacidad de utilizar dichos objetos internos, a los efectos de calmarse, consolarse y protegerse.

\section{En relación al objeto:}

d) Capacidad para vincularse emocionalmente con los demás.

e) Capacidad de pedir y aceptar ayuda y de confiar en la bondad de los objetos externos.

f) Capacidad de distanciarse y despedirse.

En las perturbaciones estructurales encontramos fallas y limitaciones en algunas de estas funciones.

Por lo demás, cabe aclarar que en estas perturbaciones hay una interrelación entre aquellas capacidades que están en déficit, de modo tal que algunas son determinantes para el surgimiento de las otras. Así, por ejemplo, las atribuciones disfuncionales respecto de las intenciones ajenas -de rechazo, hostilidad, desprecio, etc.- (capacidades de aprehensión y pensamiento, f), vividas en modo de equivalencia psíquica (esto es, con un grado de certidumbre subjetiva que no deja lugar para la duda ni el cuestionamiento), posiblemente producirán el desarrollo de un afecto hiperintenso, que será difícil de regular (capacidades de regulación, b), con la subsiguiente posibilidad de pasajes al acto y perturbaciones relacionales (capacidades de regulación, e) (ver el ejemplo clínico).

De este modo, la capacidad para proteger la relación de los propios impulsos y afectos (capacidades de regulación, e) se ve perturbada debido a la falla en la regulación emocional (capacidades de regulación, b), derivada, a su vez, de las atribuciones disfuncionales (capacidades de aprehensión y pensamiento, f) vividas en modo de equivalencia psíquica. 
Cabe subrayar la importancia del establecimiento de tales relaciones, así como de la determinación de cuáles son las fallas prioritarias y cuáles las derivadas, en la evaluación del paciente, a los efectos de establecer focos de trabajo.

Entendemos en primer término por focos las áreas problemáticas específicas que son significativas para las perturbaciones psicodinámicas del paciente (especialmente conflictos, patrones vinculares, vulnerabilidades estructurales) (Dagnino Robles, 2012). De ahí que sobre ellos han de centrarse las intervenciones específicas, que diferirán en función del tipo de foco elegido, como se verá a lo largo del presente trabajo.

\section{Niveles de la estructura}

Tanto en la estructura como un todo, como en cada una de las capacidades que la componen, es posible establecer un gradiente en cuanto a su nivel de integración. Distinguimos entonces cuatro niveles: nivel alto de integración, nivel medio, nivel bajo, nivel desintegrado.

Dada la amplitud del tema, ilustraré este concepto con sólo una de las capacidades mencionadas más arriba, y exclusivamente en su relación con el self: capacidades de aprehensión y pensamiento, a) capacidad para formar una imagen realista de uno mismo, para aprehender los propios estados mentales y reflexionar sobre ellos.

Nivel alto de integración: "Es posible una percepción diferenciada y reflexiva de uno mismo. La imagen es básicamente constante y coherente en el tiempo. Los sucesos intrapsíquicos pueden ser observados con interés y percibidos en relación con sus correspondientes afectos. El paciente puede nombrar características y capacidades (ya sea espontáneamente o al ser preguntado) que ilustran la imagen que tiene de sí mismo, “qué tipo de persona es' y qué lo diferencia de otras personas" (Grupo de trabajo OPD, 2008, pp. 297-298).

Nivel medio de integración: "La percepción reflexiva del self es limitada. Se orienta fundamentalmente al yo que actúa. La introspección con relación a los propios afectos está reducida. La coherencia de la autoimagen se pone en duda en momentos de estrés. La diferenciación entre lo propio y lo de otros es difícil" (p. 298).

Nivel bajo de integración: "La autopercepción reflexiva, en la medida que es posible, no suscita una imagen individual reconocible. Así, los propios afectos sólo pueden ser percibidos de manera poco diferenciada; la vivencia de sí mismo está determinada por afectos negativos que se expresan sobre todo como menosprecio y rechazo. En lugar de las vivencias afectivas pueden surgir el vacío y la alienación” (p. 299).

Nivel desintegrado: "Se mantiene la incapacidad del paciente para caracterizarse a sí mismo. No surge una imagen de su personalidad con aspectos físicos, sociales y sexuales de identidad. En su lugar se describen ya sea aspectos altamente normativos, y, por lo tanto, no creíbles, o aspectos extraños y chocantes o con rasgos de grandiosidad exagerada" (p. 300).

Como vemos, la determinación del nivel estructural ilustra el grado de buen funcionamiento o de déficit de las distintas capacidades estructurales, por lo que correlacionan con los tipos de desenlaces patológicos. De este modo, el nivel alto y medio se encuentra habitualmente en los pacientes neuróticos, el nivel bajo en pacientes con trastornos de la personalidad (como los pacientes con trastorno límite de la personalidad [TLP]), mientras que el nivel desintegrado suele encontrarse en restituciones que han tenido lugar luego de un episodio psicótico.

\section{Las perturbaciones estructurales}

Estas perturbaciones deben diferenciarse de aquellas cuyas manifestaciones (síntomas, patrones vinculares) se deben a la presencia de conflictos psíquicos, ya que se ponen en juego -en un caso y en otro- aspectos diferentes de la personalidad, procesos diversos, causas precipitantes distintas y mecanismos patógenos distintos, que requieren, por tanto, abordajes clínicos también diferentes (Grande, 2002, 2007; Rudolf, 2002b, 2004, 2010). 
Si miramos las cosas desde el punto de vista del conflicto, podríamos decir que cuando se infiere la presencia de un conflicto psíquico tras las manifestaciones sintomáticas, el interés se centra en los contenidos ocultos (deseos y temores) y las defensas vinculadas con ellos. Las manifestaciones mencionadas se suponen expresión de procesos inconscientes, cuya causa son.

En cambio, la búsqueda de tales significados ocultos no tiene sentido cuando predomina la perturbación estructural, y sería un error tomar por ese camino partiendo de las manifestaciones clínicas, las que obedecen - en este caso - a las limitaciones del self para disponer de las capacidades estructurales y no consisten en un derivado de algo reprimido.

En una primera caracterización de las mismas, se podría decir que en ellas se halla limitada la accesibilidad de aquellas funciones psíquicas -reseñadas en capacidades de aprehensión y pensamiento - que son necesarias para la organización y regulación del self, como así también para su relación con los objetos internos y externos (por ejemplo, dificultades para aprehender los estados mentales ajenos y empatizar con ellos, lo que produce una serie de desencuentros y problemas vinculares de diversa índole).

Esta limitación es producto de perturbaciones tempranas en el vínculo del niño con sus cuidadores, las que tienen como consecuencia una restricción en la maduración de dichas funciones, o, lo que es lo mismo, la prevalencia de niveles bajos de integración estructural. Esto último implica que dichas funciones son poco confiables y vulnerables, esto es, que muestran un modo de funcionamiento cualitativamente diferente al que poseen cuando hay un buen nivel de integración.

Podríamos decir que el adulto que posee un buen nivel estructural ha logrado un proceso de desarrollo en el que se evidenció una progresiva diferenciación e integración, cuyo resultado se muestra -entre otras cosas- en la creación de un espacio psíquico del que puede disponer para tramitar en él los diversos conflictos que lo perturban (Rudolf, 2002a).

Por el contrario, en el trastorno estructural ha habido un déficit del desarrollo, por lo que no se han logrado ciertas diferenciaciones estructurales y fases del proceso de integración. De ahí que el self no tenga a su disposición dicho espacio psíquico, no pueda regularse adecuadamente, ni sea capaz de establecer relaciones interpersonales satisfactorias (Grupo de trabajo OPD-2, 2008; Rudolf, 2002a).

Podríamos decir también que en estos casos el paciente pierde - en situaciones de estrés - la capacidad de que su self se ubique como agente y que posea un sentimiento de autoeficacia. Por este motivo - desde su perspectiva - su padecer y las diversas crisis emocionales y vinculares por las que atraviesa obedecen al comportamiento inadecuado (rechazante, incomprensivo, hostil, atemorizante, etc.) del otro, que resulta perturbador y difícil de resistir, lo cual lo compele a actuaciones, a ataques furiosos contra el mundo objetal, a la huída y la retracción, a acciones forzadas hacia sí mismo que habitualmente tienen un carácter agresivo, etc. (Rudolf, 2004, 2013).

Así las cosas, encontramos un cuadro clínico que deriva de diversas carencias y perturbaciones en las capacidades estructurales de aprehensión y pensamiento en las que se manifiesta también una cierta forma de déficit en el sentimiento del self como agente.

\section{EI conflicto psíquico}

La palabra viene del latín (conflictus, confligere: colisión, antagonismo) y se refiere a la colisión entre distintos elementos en el interior de una persona, o entre una persona y otra.

La conceptualización del mismo atraviesa toda la obra de Freud y es considerado como "el eje definidor del psicoanálisis" (Smith, 2003, p. 49).

Es importante distinguir entre conflictos externos y conflictos internos. A su vez, estos últimos han de diferenciarse en conscientes e inconscientes. "La experiencia muestra que precisamente los conflictos internos $e$ inconscientes juegan un rol predominante en el surgimiento de las perturbaciones neuróticas" (Mentzos, 2017, p. 75). Esta afirmación implica que no es el conflicto per se el que determina un desenlace patológico, sino su carácter de inconsciente, en tanto ha sufrido la acción de una defensa patógena (Schüssler et al. 2006).

Podemos considerar que existen los siguientes frentes de conflicto: 
- Del yo con el superyó

- Del yo con el mundo exterior (el otro, conflicto interpersonal)

- Del yo con las pulsiones y con los afectos

- De distintas pulsiones (o sistemas motivacionales) entre sí (Grupo de trabajo OPD-2, 2008).

\section{La estructura propiamente dicha y el conflicto}

A los efectos de este trabajo tomaré en consideración las relaciones y contrastes entre el concepto de estructura y el de conflicto. Llevaré a cabo dicha comparación en base a tres aspectos, sin pretender agotar la complejidad del tema (Stass, Grande, Janssen, Oberbracht y Rudolf, 2014).

\section{Reactivación del conflicto o de la vulnerabilidad estructural}

El primero de ellos tiene que ver con el modo en que se desencadena el padecimiento según sea que lo que se reactiva es un conflicto intrapsíquico, o que aquello que se pone en juego es la reactivación de una vulnerabilidad estructural (o de varias) que se hallaba compensada hasta ese momento.

En el primer caso suele tratarse de una situación a la que se enlaza por algún motivo un deseo presente en el paciente - posiblemente desde su infancia - de modo inconsciente y que se halla enlazado a la ansiedad o a la culpa que despierta, así como a la correspondiente defensa. La situación en cuestión representa, sea una satisfacción (Freud, 1916/1979), sea una frustración -interna o externa- de dicho deseo (Freud, 1912/1980b; Stass et al., 2014).

Como ejemplo de la primera de estas alternativas (satisfacción del deseo), cabe hacer referencia a un paciente de 35 años que se desempeñaba exitosamente en una empresa. A lo largo de más de 10 años fue considerado como un lúcido colaborador y un trabajador infatigable, lo que hizo que fuera ascendiendo desde un modesto cargo en sus inicios, hasta una posición de sub-gerente. Pocos meses después de este último ascenso, el gerente, de 56 años de edad, que se encontraba en un cargo superior al del paciente en el escalafón de la empresa, debió retirarse de la misma debido a un accidente cardiovascular que casi le cuesta la vida. Así las cosas, le fue ofrecido dicho puesto al paciente, quien lo aceptó con el mayor entusiasmo.

No obstante, poco después de instalarse en el nuevo lugar, comenzó a perder la motivación que lo había impulsado durante los años anteriores, se volvió taciturno y descontento con su trabajo, y comenzó a cometer una serie de torpezas en el mismo que llegaron a poner en serio riesgo su permanencia en la firma. Fue en ese estado que decidió consultar.

El trabajo analítico descubrió que el ascenso en el cargo mediante el reemplazo de su superior, tras la enfermedad que este había padecido, representó para él ocupar el lugar del padre, por vía de la satisfacción de un deseo parricida (parcialmente consumado en los hechos, en la figura del gerente) que abrigaba desde la infancia, unido a deseos ambiciosos y de superación de la figura paterna. La culpa por dicha realización simbólica de su deseo (ya que consideró, inconscientemente, que la enfermedad del gerente era producto de su propio deseo de sustituirlo), derivó en las alteraciones de su estado anímico y en su mal desempeño laboral, cuya intencionalidad última era promover su despido de la empresa y tener que abandonar la misma, tal como hizo su jefe, en lo que podía conjeturarse la realización de un castigo por los deseos mencionados.

En el caso de las perturbaciones a predominio de déficit estructural, la situación desencadenante es muy diferente. En ella, el futuro paciente se encuentra ante una tarea que desborda sus capacidades de elaborarla y que pone al descubierto vulnerabilidades y déficits estructurales que se hallaban hasta ese momento compensados por la situación previa.

Encontramos dos clases de situaciones prototípicas: aquellas que tienen que ver con el crecimiento y las distintas tareas que la vida impone (salir de la casa de los padres, ingreso en el mundo laboral, establecimiento de una relación de pareja, nacimiento y crianza de los hijos, etc.). En cada uno de estos casos el sujeto debe echar mano de diversas capacidades estructurales que, si se encuentran en falta, determinan el fracaso en la tarea de que se trate (Rudolf, 2013). 
La otra clase de situaciones es aquella en la que un trauma, una pérdida, una migración o un evento que cambia por completo las coordenadas con las que se manejaba el sujeto hasta ese momento, dejan en evidencia carencias estructurales que se hallaban compensadas en su situación anterior.

Así, una paciente de 50 años, que consultó en un estado de angustia y de notoria desorientación vital, refirió que hacía pocos meses había muerto el hombre con quien vivía. El trabajo analítico mostró que la paciente había conocido a quien después fuera su pareja haciendo una práctica de meditación en la institución que este dirigía. Comenzó siendo su alumna, posteriormente fue su ayudante hasta terminar siendo su compañera. Adoptó su técnica y la teoría que la sustentaba, colaboró con él en todo lo referido a la institución y trabajó con los pacientes que él le derivaba. Según sus propias palabras, con él "formaban un todo".

La relación con este hombre, a quien había ubicado en el lugar de ideal y cuyas ideas había adoptado hasta el detalle, así como las sugerencias que él le hiciera respecto a su desarrollo profesional y a otras cuestiones, mostraba que gracias a dicha relación suplía la falla en cuanto a la internalización de una formación estructural (el ideal del yo) que le hubiera permitido tener sus propios objetivos e ideales, seguir, por así decir, su propio camino y no uno trazado por su pareja.

De igual forma, la relación con el mismo tenía la función de hacer las veces de prótesis de lo que podríamos llamar su self incompleto (Helman, 1992), de modo tal que mediante una identificación de carácter fusional con él, se sentía "parte de un todo", segura de sí misma y con un equilibrio en el sentimiento de estima de sí.

La pérdida de la relación constituyó entonces una verdadera catástrofe narcisista, que puso al desnudo la fragilidad de su self y la falta de estructuras internas que le hicieran de un faro en la vida.

Como vemos, entonces, el tipo de situaciones desencadenantes, sea de una patología determinada por el conflicto, sea de una perturbación a predominio de déficit estructural, es diferente, tanto en su fisonomía fenoménica como, primordialmente, en la dinámica psicológica que entraña.

Por lo demás, vale la pena reiterar que en tanto en las perturbaciones estructurales el paciente suele adjudicar, habitualmente, la sobrecarga relacionada con el surgimiento de sus padecimientos a factores externos (actitudes perturbadoras de la pareja, exigencias laborales excesivas, pérdidas, etc.), se corre el riesgo de entender dichos padecimientos como meramente "reactivos" al impacto de dichos factores, en cuyo caso permanece oculta la dinámica responsable de los mismos.

De este modo, el malestar de la paciente de 50 años mencionada, podría ser comprendido como efecto del trauma consistente en la pérdida de su pareja, con lo cual quedarían desatendidas las vulnerabilidades estructurales reactivadas por dicha pérdida y no serían incluidas como foco de un trabajo terapéutico.

\section{Relación entre el nivel estructural y la madurez del conflicto}

El segundo aspecto alude al hecho de que cuanto más integrado es el nivel estructural, más habitualmente nos encontramos con conflictos "maduros", como el conflicto edípico, por ejemplo. Por el contrario, cuanto menos integrado es el nivel de la estructura, más arcaicos son los sistemas motivacionales que intervienen en el conflicto (dependencia-autonomía, sistema de autovaloración, etc.), como así también las defensas implicadas, que tienden a activarse en el terreno interpersonal y no tanto en el intrapsíquico (proyección, desmentida, etc.) (Rudolf, 2013).

Asimismo, cuanto mejor es el nivel estructural mayores son los recursos para el trabajo con los conflictos, lo que posee la mayor importancia para el abordaje de los mismos, ya que es necesario contar con un buen nivel estructural para que pueda tener lugar el abordaje interpretativo de aquellos y su posterior elaboración por parte del paciente.

\section{Casos mixtos}

El tercer aspecto tiene que ver con esos casos mixtos en los que si bien el conflicto y sus derivados se encuentran en primer plano, los déficits estructurales que subyacen al mismo determinan la intensidad de dicho conflicto y su eventual inaccesibilidad terapéutica. 
Podríamos ejemplificar esta alternativa con el caso de una joven de 18 años, que actúa sus intensos celos contra la segunda esposa del padre, haciéndoles violentas escenas, tanto a ella como a su progenitor, cuando se encuentra con ellos.

La paciente reclama prioridad en la atención del padre, tanto para ella como para su madre y hermano, mientras que dice que la segunda esposa y los nuevos hijos (que esta ha tenido con el padre) deberían ocupar un lugar secundario.

La intensidad de los reclamos -y de los afectos con ellos asociados- no ceden ante la interpretación del conflicto edípico (ella se coloca en el lugar de la esposa despechada), que se mantiene estable e inalterado.

La situación cambia cuando el terapeuta lleva a cabo una modificación en su estrategia y pone en primer plano la consideración de los déficits estructurales que se encuentran en el trasfondo del cuadro clínico, que había considerado como una histeria. Entre ellos cabe mencionar: 1) la falla en la regulación de los afectos, que se traducen entonces en el despliegue motriz que lleva a las escenas mencionadas; 2) la inalterable convicción de tener razón en sus reclamos (funcionamiento en equivalencia psíquica); 3) una fuerte dependencia del padre, vivido como objeto sostén de su narcisismo, razón por la cual el ser postergada es vivido como una insoportable herida narcisista.

Se vuelve comprensible entonces el hecho de que la eficacia de estas perturbaciones en el nivel de la estructura - que permanecían sin ser abordadas mientras las intervenciones focalizaban en el conflicto edípico-sea la responsable, tanto de la magnitud e intensidad del conflicto, como de su carácter de inamovible e inalterable.

Es sólo mediante un cambio en la estrategia clínica, que pone entonces en primer plano el trabajo con las perturbaciones estructurales mencionadas, que es posible avanzar en el trabajo psicoterapéutico.

Cabe agregar que hay diversas clases de cuadros mixtos, entre los cuales poseen importancia no menor aquellas formas neuróticas que en ciertas ocasiones hacen un pasaje a un funcionamiento fronterizo, por vía de falla estructural puntual. He analizado con detalle -en otra ocasión- un caso en el que tiene lugar este pasaje (Lanza Castelli, 2016a).

\section{El enfoque terapéutico}

Cuando adoptamos invariablemente una estrategia orientada a los conflictos (quizás debido a nuestra formación, por ejemplo) cabe la posibilidad de que aparezcan una serie de dificultades en la terapia que pueden derivar de déficits estructurales que son más predominantes de lo que se asumió en un principio y que hacen que sea difícil lidiar con la dinámica del conflicto-como en el último ejemplo. Por esa razón es necesario establecer, en primer lugar y antes de iniciar el tratamiento, el peso relativo de los trastornos estructurales y de los conflictos, en los padecimientos del paciente.

A partir de esta evaluación, se pueden deducir estrategias de acción terapéutica, que estén, según el caso, predominantemente dirigidas al trabajo con los conflictos, o a lidiar con los déficits estructurales.

En dichas estrategias diferenciamos los siguientes aspectos: el encuadre, la actitud terapéutica, la jerarquización de los focos elegidos, la elaboración de los patrones relacionales, las intervenciones (Rudolf y Grande, 2006).

No obstante, antes de entrar en la caracterización diferencial de cada uno de estos elementos para la psicoterapia orientada al conflicto, la orientada a la estructura y los casos mixtos, será de utilidad llevar a cabo una caracterización general -aunque esquemática- de los dos primeros tipos de psicoterapia.

\section{La psicoterapia orientada al conflicto}

En este caso, las perturbaciones que se abordan poseen un grado alto o medio de nivel estructural y se deben a la eficacia de los conflictos. Para expresarlo mejor, podríamos decir que lo eficaz no son tanto los conflictos, sino la forma patológica de su tramitación, ya que los mismos son un fenómeno universal que encontramos también en el territorio de la salud.

La perturbación de que se trate debe ser comprendida como el resultado de una intención inconsciente del 
paciente, un deseo como derivado de una moción pulsional (en el enfoque de Freud), que debido a su colisión con el mundo exterior o con el superyó (conflicto), sufre la represión y es alejado de la conciencia y de su acceso a la motilidad. En un segundo momento, debido al fracaso de la represión, emprende el camino hacia aquellos territorios de los que fue alejado, utilizando las vías creadas por otras defensas: la proyección en el caso de la fobia, la identificación y la conversión en el caso de la histeria, la formación reactiva, la anulación, el aislamiento, etc. en el caso de la neurosis obsesiva.

En este tipo de psicoterapia se trata, entonces, de favorecer que el conflicto se tramite y se resuelva de un modo diferente. En primer término se favorecerá el devenir consciente de la moción inconsciente, por medio de la interpretación. En segundo lugar se buscará una mejor tramitación de la misma, no ya por vía de la represión (que transcurre de modo inconsciente) sino del juicio de condenación consciente. Dicho juicio permite el mantenimiento del deseo en el territorio consciente y decide acerca del grado en que se le permitirá la satisfacción en función de los valores del sujeto (ideal del yo) y de las oportunidades que ofrece la realidad (Freud, 1909/1980a, p. 116).

\section{La psicoterapia orientada a la estructura}

Esta forma de psicoterapia surge a fines del siglo XX, de la mano de Gerd Rudolf y colaboradores. Consiste en una modificación del enfoque psicoanalítico clásico y ha sido diseñada especialmente para el trabajo con aquellos pacientes cuyo padecimiento no obedece en primer término a la presencia de conflictos inconscientes, sino a la eficacia de déficits y limitaciones en su estructura psíquica. Estos pacientes han sido descriptos en la literatura como "difíciles", con "organizaciones duales", "preedípicos", con "personalidades primitivas", etc. (Cierpka, 2004) y la experiencia ha demostrado que se benefician en muy escasa medida de la técnica psicoanalítica clásica, con su énfasis en la interpretación y en el análisis de la transferencia. Manfred Cierpka sintetiza esta idea en la siguiente frase: "Cuanto más acentuados son los problemas estructurales de los pacientes, tanto más difícil resulta alcanzar el éxito con la ayuda de la técnica psicoanalítica standard" (2004, Prefacio, p. VIII).

Otros autores han ido más allá, al sostener que la aplicación de esta técnica -con dichos pacientes- puede ser perjudicial, en la medida en que las interpretaciones son vividas como críticas o ataques, o favorecen que el paciente ubique al terapeuta en el rol de una madre omnisciente (Bruch, 1973, Lecours, 2007, Rudolf, 2007).

Por su parte, la terapia centrada en la estructura tiene como pretensión y objetivo trabajar provechosamente con estos pacientes. Para ello se basa en una detallada conceptualización de la estructura psíquica (Rudolf, 2004, 2013), en la utilización de instrumentos diagnósticos eficaces para caracterizarla con precisión (Grupo de trabajo OPD-2, 2008) y en la utilización de una serie de técnicas y procedimientos encaminados a remediar los déficits y limitaciones de dicha estructura (Rudolf, 2002b, 2004, 2010, 2013).

En lo que sigue, me atengo fundamentalmente a los lineamientos propuestos por Rudolf y Grande (Rudolf, 2002b, 2004, 2010, 2013; Grande, 2002, 2007; Rudolf y Grande, 2006), agregando algunos aportes personales, pero sin hacer lugar, de un modo extenso y detallado a mis propias opiniones al respecto, en parte concordantes y en parte divergentes o complementarias con las de estos autores, lo que alargaría de un modo excesivo el presente texto.

\section{Estrategias en la psicoterapia orientada al conflicto}

\section{Encuadre}

Según la indicación de que se trate, el tratamiento puede llevarse a cabo en el contexto del psicoanálisis, que promueve la regresión del paciente, o en el contexto de la psicoterapia psicoanalítica, que pone límites a dicha regresión y tiende a trabajar con el concepto de foco (Rudolf y Grande, 2006).

En la medida en que el paciente (en el que predomina el conflicto) es fundamentalmente responsable de sus 
acciones, debido a su buen funcionamiento estructural, el encuadre terapéutico no ha de tener una tarea principalmente estructurante, que ponga límites o de apoyo, del modo en que resulta necesario cuando hay déficits en el funcionamiento de las capacidades estructurales.

Su función consistirá en definir las condiciones marco bajo las cuales los participantes aceptan que la terapia ha de realizarse (asociación libre y atención libremente flotante, frecuencia y duración de las sesiones, lugar en que se llevarán a cabo, posición cara a cara o con diván, roles de cada uno de los participantes, etc.).

Las eventuales transgresiones del encuadre (llegadas tarde, ausencias reiteradas, no acatamiento del rol, etc.) han de ser comprendidas como debidas a la acción de las resistencias o de la actualización transferencial de los conflictos inconscientes.

\section{Actitud terapéutica}

El terapeuta puede asumir que el paciente realiza una transferencia sobre él de sus mociones conflictivas inconscientes, así como de las defensas que sobre ellas recaen.

Por esta razón, prestará atención a su propia contratransferencia como una de las vías para inferir aquello que le ha sido transferido.

Una intervención terapéutica importante consistirá, entonces, en hacer conscientes para el paciente las experiencias inconscientes transferidas, por vía de la interpretación.

En este contexto encontramos un supuesto fundamental, consistente en que el paciente puede responsabilizarse de sus acciones, una vez que ha hecho consciente lo inconsciente y lo ha reconocido dentro de sí.

Por lo tanto, el trabajo terapéutico se lleva a cabo desde una posición de abstinencia benévola, que no busca proteger al paciente con actitudes de apoyo y sostén.

\section{Jerarquización de focos}

Es necesario formular una hipótesis sobre cuál de los conflictos constituye el núcleo de la perturbación y qué otros conflictos están apuntalados en él, o de él se derivan, ya que es habitual que encontremos estas interrelaciones de conflictos.

El trabajo de desvelamiento de los conflictos puede comenzar por el conflicto apuntalado, para abordar con posterioridad el conflicto central. A su vez, este último será enfocado primeramente en su vertiente defensiva, tras lo cual podrán tener lugar las intervenciones referidas a las intenciones inconscientes sobre las que recaen las defensas.

\section{Elaboración de los patrones relacionales}

El terapeuta busca identificar qué deseos y temores vinculares dirige el paciente, tanto hacia él como hacia los demás. Construye una hipótesis acerca de cómo pueden ser comprendidas como formaciones de compromiso las propuestas relacionales del paciente, a la vez que detecta en su contratransferencia sentimientos e impulsos activados por este.

Examina, junto con el paciente, los temores de este último en relación con los demás, así como las medidas que utiliza para protegerse. Señala el aspecto disfuncional de estas últimas, así como la probabilidad de que actúen como profecías que se autorrealizan, de modo tal que los temores se concreticen y los deseos relacionales queden insatisfechos.

De igual forma, busca mostrarle qué sentimientos e impulsos podría desencadenar o provocar en los demás - y en el terapeuta mismo - con dichas medidas, así como las consecuencias vinculares que tal efecto podría verosímilmente tener. 


\section{Intervenciones}

Dado que los síntomas del paciente y los problemas relacionados con los mismos son desencadenados por características situacionales que tienen un significado específico y personal, determinado por los conflictos inconscientes, se hacen necesarias intervenciones como el señalamiento, la confrontación y la interpretación, con la intención de remover resistencias y defensas, y hacer conscientelo inconsciente (Greenson, 1976).

En el análisis de larga duración pueden emplearse técnicas y estrategias que favorezcan la regresión y la activación de la transferencia.

\section{Estrategias en la psicoterapia orientada a la estructura}

Las perturbaciones estructurales se caracterizan por la limitada disponibilidad de funciones estructurales (p. ej., autoreflexión y diferenciación de los afectos, percepción realista de los objetos y empatía, autocontrol y defensas maduras, comunicación emocional e internalización de relaciones, etc.).

El nivel general de la estructura se encuentra en estos casos en el rango inferior (nivel medio a nivel bajo de integración, nivel bajo a nivel desintegrado). Los conflictos son reconocibles, pero son polifacéticos y no están claramente configurados de modo específico.

Para la comprensión de la psicodinámica en juego, es decisivo que las dificultades del paciente no sean atribuidas a intenciones inconscientes (deseos, defensas), sino que sean entendidas como consecuencia de restricciones y perturbaciones en la autorregulación y en el establecimiento de vínculos interpersonales.

Por lo demás, en el manejo de sus limitaciones estructurales no es infrecuente que el consultante haga uso de comportamientos disfuncionales que pueden tener un carácter de autodaño (por ejemplo, ante la falla en la regulación emocional, puede echar mano de comportamientos autolesivos, como cortarse, para lograr dicha regulación).

Por esa razón, en el tratamiento terapéutico de estos pacientes es de vital importancia distinguir entre sus incapacidades estructurales, por un lado, y las posibilidades, aún disponibles, de un manejo secundario de las mismas, que puede tener un carácter constructivo o destructivo.

\section{Encuadre}

Cuanto más grave sea la perturbación estructural, tanto más puede ser necesario proporcionar una estructuración externa, así como medidas que estimulen el desarrollo del aprendizaje social (por ejemplo, actividades de capacitación y recreativas).

En las psicoterapias ambulatorias se debe ofrecer una estructura estable (disponibilidad confiable del terapeuta, tanto dentro de la sesión como en el período entre sesiones).

La forma externa del tratamiento y el contenido de la terapia deben ser discutidos cuidadosamente y acordados con el paciente. Los puntos más relevantes se pueden regular mediante «contratos» (por ejemplo, con respecto al comportamiento suicida, comportamiento alimentario, comportamiento adictivo y otras formas de autolesión).

Todas las condiciones externas que dan lugar a la estructura mencionada deben estar incluidas en la relación terapéutica y ser representadas por el terapeuta (o un equipo terapéutico), de modo tal que las funciones proporcionadas (por ejemplo, gestión del cuidado, puesta de límites, contención de afectos difíciles) puedan ser progresivamente adoptadas o apropiadas por el paciente como una capacidad estructural (por ejemplo, como puesta de límites, tolerancia del afecto).

Las transgresiones eventuales de las reglas no se indagan con vistas a las intenciones ocultas del paciente, sino que se responden con límites y firmeza. 


\section{Actitud terapéutica}

En vista de los habituales comportamientos relacionales devaluatorios y destructivos del paciente, es necesario construir, en primer lugar, una actitud terapéutica que contribuya a que la relación terapéutica y el trabajo conjunto no se vuelvan imposibles ni sean arruinados.

A esta actitud pertenece, principalmente en los comienzos de la terapia, una posición parental, que lleva al terapeuta a asumir una función de contención, de yo-auxiliar, de ayuda, de ocuparse en poner a disposición del paciente su propia percepción y vida emocional, de modo que hagan las veces de reflejo de lo que ocurre en el paciente, y también a establecer límites firmes cuando tal cosa sea necesaria.

También es importante señalar al paciente las oportunidades que tiene disponibles para afrontar sus dificultades estructurales, de una manera más constructiva.

Pero esto implica que el terapeuta no considere el comportamiento del paciente como una transferencia dirigida hacia él, en sentido personal, sino que proponga enfocar dicho comportamiento - preferentemente junto con el paciente - como un patrón problemático de actuar y vivenciar, que este ha de aprender a manejar.

\section{Jerarquización de focos}

Inicialmente es necesario establecer en cuáles funciones estructurales el paciente necesita principalmente ayuda terapéutica, a los efectos de poder regularse a sí mismo y a sus vínculos. A este respecto es válido considerar que cuanto más profunda es la perturbación estructural, tanto más se ha de poner el acento en los aspectos del self descriptos más arriba (capacidades de aprehensión y pensamiento, a) autopercepción; capacidades de regulación a, b) autocontrol). En este caso, la tarea del terapeuta consistirá en promover la autorrelación y la autonomía del self.

Los pacientes de este tipo a menudo encuentran que las relaciones con los demás mejoran sin ayuda adicional, una vez que han adquirido mayor orientación y fuerza en sí mismos.

Asimismo, en este nivel de perturbación, devienen centrales las carencias o fallas en la dimensión del vínculo (internalización - capacidades de vínculo, a-, pérdida - capacidades de vínculo, d-).

La mejoría generalmente se logra sólo de manera imperfecta por medio del contenido de las palabras, por lo que en tales casos la actitud y el comportamiento del terapeuta (por ejemplo, la confiabilidad, la solidez, la benevolencia) son más importantes para el efecto terapéutico que el contenido (mental) del intercambio.

En aquellos cuya perturbación estructural es menor, ha de ponerse en primer plano la relación con los demás (percepción del objeto - capacidades de aprehensión y pensamiento, c-, comunicación — capacidades de comunicación, c, d-, separación - capacidades de vínculo, d-), sobre la cual se focalizará el trabajo terapéutico.

Por su parte, el terapeuta podrá promover dicho trabajo presentándose más claramente como un otro, con un punto de vista diferente al del paciente (Rudolf, 2013).

\section{Elaboración de los patrones relacionales disfuncionales}

El terapeuta establece, junto con el paciente, qué dificultades, limitaciones y vulnerabilidades estructurales inciden en su comportamiento relacional.

No relaciona las propuestas relacionales problemáticas del paciente con su persona, sino que se esfuerza por identificar una configuración relacional en la que el problema vincular que surge en el contacto terapéutico se objetive y se explore, por así decir, lado a lado con el paciente.

El terapeuta ayuda al paciente a controlar su comportamiento relacional, con el conocimiento adquirido en dicha exploración. 
Especialmente en el caso de comportamientos agresivos y destructivos, puede resultar de utilidad la representación de que los mismos son, por así decir, expresiones de un "ello", esto es, de acciones o fuerzas aún no integradas y por tanto, difíciles de regular.

Esta representación puede ayudar al paciente a que se diferencie de su comportamiento y se distancie de él. De lo contrario, sería difícil no experimentar al paciente - y que este se experimente a su vez - como intencionalmente destructivo.

Lo que también es importante en este punto es la consideración de que los pacientes con limitaciones estructurales pueden desarrollar - de forma secundaria - estrategias de afrontamiento que poseen un carácter agresivo, desvalorizante o destructivo.

Aparecen entonces (parcialmente) como actores responsables y deben, por lo tanto, ser abordados como los autores de este afrontamiento disfuncional: no se trata del "ello", en ese caso, sino del paciente mismo.

Por lo demás, los patrones disfuncionales en cuya base se encuentran perturbaciones estructurales no son, en modo alguno, siempre agresivos, sino que a menudo tienen un carácter defensivo o evitativo (Grande, 2007).

Muchas veces la retracción o los controles rígidos de las relaciones sirven como defensa respecto a susceptibilidades determinadas estructuralmente (por ejemplo, una identidad frágil, un sentimiento de sí menoscabado, etc.).

En estos casos el terapeuta trabaja dichas vulnerabilidades, junto con el paciente, tratando de traducirlas en palabras, mientras pone particular interés en identificar en qué situaciones y vínculos específicos las mismas se activan.

El terapeuta acepta y connota positivamente el modo en que el paciente protege sus vulnerabilidades mediante determinados modos vinculares. De este modo, resaltan con claridad sus competencias para el afrontamiento.

En un momento posterior, puede tornarse más claro que esta autoprotección tiene también un costo importante para el paciente, por ejemplo que los deseos relacionales sólo puedan satisfacerse en un modo muy reducido, o no se satisfacen en lo absoluto.

\section{Intervenciones}

Cuanto más grave es el trastorno estructural, tanto más precaución y moderación se requiere con respecto a las técnicas de movilización afectiva y tanto menos se promoverán la regresión y la dependencia. En su lugar, se utilizarán técnicas que permitan una estabilización basal del self, mientras que los aspectos interaccionales referidos a los objetos, serán abordados en un momento posterior de la terapia

Por lo demás, cabe consignar que los procedimientos no verbales son a menudo más adecuados que las intervenciones lingüísticas, para proporcionar al paciente un sentido auténtico de su propia persona.

En la medida en que las restricciones estructurales se manifiestan a través de sobrecargas no específicas (Cf. Reactivación del conflicto o de la vulnerabilidad estructural) y no, como los conflictos neuróticos, mediante situaciones con contenido simbólico, las interpretaciones sobre factores inconscientes desempeñan en estos casos un papel menor.

La mirada se centra en el cómo (por ejemplo, objetivando y diferenciando las restricciones estructurales) y no en el por qué, ya que no se busca algo oculto, sino que se ayuda al paciente a que gane objetividad y distancia respecto de sus dificultades y que aprenda a controlarlas.

En lo que hace a los abordajes específicos para los déficits particulares (diferenciación de los afectos, aprehensión del estado mental ajeno, regulación emocional, etc.), su número es variado y extenso, por lo que solo serán incluidos de modo parcial en el análisis del material clínico (Cf. Grande, 2002, 2007; Rudolf, 2002b, 2004, 2010, 2013).

\section{Estrategias en la psicoterapia de perturbaciones determinadas por el conflicto y complicadas por la presencia de perturbaciones estructurales}

En estos casos encontramos, según Rudolf $(2010,2013)$ y Rudolf y Grande (2006), en primer plano la eficacia de determinados conflictos. No obstante, una evaluación cuidadosa del caso nos muestra la presencia 
simultánea de determinadas perturbaciones estructurales. No es infrecuente que tales perturbaciones sean descubiertas primeramente en el curso mismo del tratamiento, en la medida en que advertimos las dificultades del paciente para beneficiarse del desvelamiento del conflicto, para aceptar una imagen diferente de sí mismo y reconciliarse con la propia situación (Cf. el ejemplo citado en Casos mixtos).

En tales casos, se deja ver que falta una base estructural estable para procesar el conflicto, por lo cual ha de promovérsela en primer término a los efectos de posibilitar la integración del mismo.

En otros casos mixtos, según he podido observar en la clínica, lo que domina el cuadro inicialmente son las perturbaciones estructurales y es solo después que estas han sido elaboradas hasta cierto punto, que se hacen visibles y abordables los conflictos. Este es el caso de la paciente presentada, más adelante, en el apartado "Ejemplo clínico".

\section{Encuadre}

No hay en estos casos una propuesta inequívoca de un tipo de encuadre, para un tratamiento que suele desarrollarse de manera ambulatoria.

Por lo demás, es habitual que tanto los pacientes como los terapeutas ubiquen los padecimientos de aquellos en el territorio de las neurosis. Por esta razón, se corre el riesgo de subestimar la contribución de las perturbaciones estructurales en el padecer del consultante y de exigirle a este más de lo que en realidad está en condiciones de dar.

Cuando tales perturbaciones son descubiertas, la estrategia debe modificarse, así como el encuadre, que se volverá más estructurado y, según la gravedad del caso, más o menos similar al mencionado en el caso anterior (Ver "Encuadre" dentro de "Estrategias en la psicoterapia orientada a la estructura")

\section{Actitud terapéutica}

Se dirige hacia la necesidad actual del paciente, esto es, hacia el tipo de problemática actual que este presenta.

Se orienta en un sentido básicamente estructural, lo cual quiere decir que el trabajo con los conflictos tiene lugar con la suposición implícita de que los pasos que se den serán relativos y que tarde o temprano será necesario habérselas con las perturbaciones estructurales, para volver, después de ello y estando el paciente mejor provisto por la atenuación de dichas perturbaciones, a enfrentarse con la problemática del conflicto.

Esto significa que la focalización inicial en los conflictos tiene lugar con reservas y sin la expectativa de que realmente se pueda encontrar una solución viable de los mismos, trabajando solo en este territorio.

\section{Jerarquización de focos}

Dado que a menudo no es posible la elaboración de los conflictos si no se cuenta con los recursos estructurales adecuados, cabe considerar, como focos terapéuticos, tanto a los conflictos como a las perturbaciones estructurales.

En el trabajo terapéutico puede resultar perturbador si, por un lado, la tramitación de los conflictos se revela infructuosa debido a limitaciones estructurales, mientras que, por otro lado, el trabajo focalizado en la estructura puede dificultarse debido a complicaciones derivadas de los conflictos (por ejemplo, el trabajo para promover la diferenciación de los afectos puede verse complicado por una sofocación defensiva de los mismos).

En caso de duda, hay que privilegiar el tema estructural. Cuando el paciente presenta un conflicto específico, es importante prestar atención al aspecto estructural implicado, así como dirigir la atención del paciente sobre dicho aspecto. 


\section{Elaboración de patrones relacionales}

Es necesario identificar cuáles perturbaciones estructurales incrementan las interacciones problemáticas y disfuncionales del paciente (por ejemplo, su falta de empatía, que produce constantes malos entendidos y frustraciones en los demás).

En ciertos casos observamos que los patrones vinculares problemáticos hacen las veces de protección respecto de vulnerabilidades estructurales (Grande, 2007), como en el caso de la paciente de 50 años mencionado en "La estructura propiamente dicha y el conflicto", quien mantenía una relación de sumisión-dependencia que neutralizaba sus temores al abandono, el cual produciría un colapso narcisista.

\section{Intervenciones}

En términos generales, cabe decir que el trabajo con los conflictos o el procesamiento de temas estructurales se realiza según los puntos de vista que han sido considerados en relación con los dos primeros tipos (ver "Estrategias en la psicoterapia orientada al conflicto" y "Estrategias en la psicoterapia orientada a la estructura").

El terapeuta debe poder dejar temporariamente de lado el trabajo con los conflictos, cuando se tornan visibles los aspectos estructurales, aún cuando el paciente ponga el acento en los primeros.

Asimismo, ha de tener en cuenta que el trabajo con las perturbaciones relacionales - en este tipo de pacientes - no debe ser un fin en sí mismo, sino que, en última instancia, debe conducir a encontrar el núcleo estructural de la dificultad y explorarla con el paciente.

\section{Ejemplo clínico}

El siguiente fragmento clínico pretende ilustrar, aunque sea brevemente, algunas de las técnicas específicas que se utilizan para el trabajo con los pacientes que padecen perturbaciones estructurales. Para ello, tomaré el comienzo de la primera entrevista con una paciente de 23 años, cuya terapia se extendió a lo largo de 3 años, con resultados satisfactorios.

Dada la falta de espacio, no llevaré a cabo un relato in extenso del tratamiento, ni abundaré en consideraciones acerca del conflicto psíquico. Baste decir que se trataba de una paciente con considerables perturbaciones estructurales, que desarrollaba relaciones de dependencia intensa con sus parejas y que pertenecía al tipo mencionado en último término (conflicto más perturbación estructural).

Comienzo de la primera entrevista (Silvina, 23 años):

T.: Bueno, contame el motivo por el que me llamaste.

P.: Primero, no me quiero nada. Autoestima cero. Físicamente muy bien, pero interiormente muy mal, me maltrato muchísimo y no tengo nada de autocontrol, en el sentido de...no con todo el mundo. Hay personas con las que lo tengo. Hay cosas que no me gustan y las aguanto. Con el chico con el que estoy ahora no pienso y sólo actúo...

T.: ¿Podés darme un ejemplo?

P.: Él me dice "hoy no puedo quedar" y yo pienso, no quiere quedar conmigo, no sé qué, no sé cuánto y ya le armo un escándalo, grito, insulto, le digo cosas terribles...y no es normal. Después le pido perdón. Soy consciente de que lo que hago está mal, pero lo vuelvo a hacer y lo vuelvo a hacer. Lo primero que me sale en ese momento, bum, no lo controlo! Quiero saber por qué me siento así, por qué siento eso, por qué se me vuelve a repetir el mismo patrón una y otra vez cuando se supone que ya soy consciente de ello. Quiero poder pensar y controlarme, pero no puedo. Necesito ayuda.

En primer término, cabe identificar las perturbaciones estructurales que encontramos en Silvina. A tal efecto, he destacado en cursiva las expresiones en las que dichas perturbaciones se manifiestan y he consignado entre paréntesis los ítems presentes, de aquellos referidos al hablar de las distintas capacidades estructurales. 
Una perturbación considerable se expresa en la frase "Él me dice 'hoy no puedo quedar' y yo pienso, no quiere quedar conmigo, no sé qué, no sé cuánto y ya le armo un escándalo, grito, insulto, le digo cosas terribles"

En la escena referida, vemos un primer momento en el que Silvina lleva a cabo una "traducción" de las expresiones de su pareja ("hoy no puedo quedar"), por vía de una atribución sesgada ("no quiere quedar conmigo"), basada en creencias disfuncionales acerca de sí misma y del otro, lo cual supone una perturbación en la capacidad de aprehender los estados mentales ajenos efectivos, de un modo plausible, descentrado y no egocéntrico (capacidades de aprehensión y pensamiento, f).

Por lo demás, dicha atribución no es vivida por la paciente como una mera idea, una conjetura o una fantasía, sino con la más alta certidumbre subjetiva, como algo que es indiscutiblemente de ese modo, o sea, en "equivalencia psíquica" (capacidades de aprehensión y pensamiento, g).

Junto con ello, y debido a su equipamiento deficitario, Silvina posee reducidas posibilidades de contrastar y corregir la atribución sesgada mencionada (Grande, 2007).

Un segundo momento de la escena muestra la reacción emocional de Silvina ante la interpretación que realizó de lo dicho por su pareja ("ya le armo un escándalo, grito, insulto, le digo cosas terribles").

Según pudimos ver con detalle durante nuestro trabajo, Silvina no podía diferenciar con claridad — cuando comenzó el tratamiento - cuáles eran los afectos presentes en su reacción emocional (capacidades de aprehensión y pensamiento, b). Poco a poco fuimos pudiendo diferenciar en dicha reacción: una angustia intensa, un pronunciado dolor anímico, un colapso narcisista, intensos celos, un marcado sentimiento de exclusión y una fuerte hostilidad (que dominaba inicialmente el cuadro).

Por otro lado, dicha reacción se traducía inmediatamente en acción, sin la menor mediación ni regulación, por lo que hacía recaer sobre su pareja una andanada de gritos e insultos. Vemos en este caso una falla en la regulación emocional (capacidades de regulación, a, b), a la vez que una merma considerable en la agencia (autoeficacia) del self, que era arrollado por los afectos mencionados, sin que pudiera tener una posición activa, de alguna índole, respecto de los mismos y de sus exteriorizaciones. En efecto, no era Silvina quien decidía activamente gritar, insultar, etc., sino que tal cosa "le acontecía".

Por otra parte, en este estado tampoco podía pensar, ya que el mismo tenía lugar en un estado de conciencia reducida.

Asimismo y por las mismas razones, en este estado mental le era imposible a la paciente anticipar cómo su reacción impactaría en su pareja (capacidades de regulación, f), o proteger la relación de su desborde emocional (capacidades de regulación, d).

Otra perturbación estructural la encontramos en la falta de internalizaciones de objetos buenos, que pudieran ayudarla a regular su emoción, a consolarse y confortarse (capacidades de vínculo, a). En su lugar, vemos la presencia de objetos hostiles que la atacan continuamente ("me maltrato muchísimo"), lo cual incide en el déficit del sentimiento de estima de sí ("no me quiero nada. Autoestima cero") (capacidades de regulación, c).

Las tendencias autopunitivas ("me maltrato muchísimo") son la forma en que aparecen los sentimientos de culpa, cuando hay un bajo nivel estructural (Grupo de trabajo OPD-2, 2008, p. 304).

Dado que el motivo de consulta estaba centrado en las dificultades para pensar y regularse ("Quiero poder pensar y controlarme, pero no puedo. Necesito ayuda") y que dichas dificultades incidían considerablemente en la relación de pareja de Silvina, que constituía el centro de sus intereses vitales en ese momento, consideré que este era un foco a privilegiar en el primer tiempo de nuestro trabajo en común.

No abundaré en este punto en temas como el encuadre, la actitud terapéutica, la alianza de trabajo, la base segura, etc. y de cómo fueron puestos en juego en el tratamiento de Silvina, ni haré mención a la evaluación completa (formulación del caso) que llevé a cabo en las primeras entrevistas, sino que solo trataré de describir algunas de las intervenciones que utilicé en relación al foco elegido. En la selección que hice de dichas intervenciones tomé en cuenta tanto las propuestas de Gerd Rudolf y Tilman Grande, como las de Allen, Fonagy y Bateman, ya que entre la terapia orientada a la estructura y la terapia basada en la mentalización encontramos, no solo solapamientos teóricos (Lanza Castelli, 2016b), sino también complementariedades prácticas.

Para mayor claridad haré referencia, a lo largo del texto, a las fuentes que correspondan en cada caso.

Vale la pena reiterar entonces que en las situaciones mencionadas, el self de Silvina se veía arrollado por los afectos y carecía de toda agencia (autoeficacia) respecto de los mismos. 
La primera intervención que puse en práctica, respecto a este estado de cosas, fue sugerirle que cuando sintiera que la ira estaba por inundarla, apretara el botón de pausa, como hacemos con un grabador o un equipo de música (Allen, Fonagy y Bateman, 2008).

La forma de apretar dicho botón consistía en implementar un monólogo interior, en el que Silvina se dijera a sí misma “¡pará!”, “¡contrólate!”, “ite estás por descontrolar, pará!” y otras verbalizaciones equivalentes, que fueron establecidas de común acuerdo con ella.

La paciente se interesó con la propuesta y comenzó a ponerla en práctica con el mejor de los resultados.

Podríamos conjeturar que la eficacia de este proceder se debió a los siguientes factores. Por un lado, porque de este modo se llevaba a cabo una co-regulación. Mi sugerencia fue tomada por ella como viniendo de alguien en quien confiaba y a quien acreditaba. El hecho de hacerla suya (en tanto proveniente de mí) le permitió recuperar en parte la posición activa y poder controlar, entonces, los desbordes y su traducción en acciones.

Por otra parte, el hecho mismo de que yo le hiciera la propuesta implicaba una invitación a que se pusiera en posición agente, invitación sostenida (en lo implícito) por una creencia de mi parte respecto a su capacidad de hacerlo, lo que supone una actitud validante, una acreditación de su persona y un respaldo, así como una narcización de su self.

En un segundo momento, y cuando la paciente ya había logrado algunos éxitos por medio de este procedimiento, le sugerí que se esforzara por identificar in statu nascendi, los primeros conatos que advertían que estaba a punto de desencadenarse el estallido emocional (Rudolf, 2013), para que pusiera en práctica esta técnica en ese preciso momento.

Asimismo, le propuse que fuera identificando qué situaciones interpersonales específicas detonaban su reacción emocional, para que pudiera advertir con antelación lo que estaba a punto de suceder (Rudolf, 2013) y apretara en ese mismo momento el botón de pausa.

El freno en su reacción que de este modo conseguía permitía que en ese lugar se habilitara un espacio mental en el que podía habitar un pensamiento. Y ese fue el siguiente objetivo. Para ello, describí ante Silvina con detalle la secuencia de las escenas que me relataba, que tenían todas un mismo formato: su pareja decía o hacía algo -ella interpretaba por vía de atribuciones propias y sesgadas la manifestación de la misma, y lo hacía en equivalencia psíquica-, de esta configuración (interpretación + equivalencia psíquica) surgía el afecto hiperintenso.

Pusimos de relieve, entonces, el hecho de que ella daba crédito absoluto a sus atribuciones en esas circunstancias, $\mathrm{y}$ le propuse que en ese momento en que ahora podía pensar, se interrogara acerca de si no había otras formas de entender lo que su pareja había dicho o hecho (Bateman, Fonagy, 2006; Grande, 2007). Le sugerí también que si la ocasión lo permitía, pusiera por escrito la interpretación que había hecho y lo que en ese momento estaba pensando, o que lo dijera en voz alta para sí misma, ya que el poner en palabras (verbalmente o por escrito) tiene un efecto regulador involuntario sobre la intensidad emocional (Lieberman, Eisenberger, Crockett, 2007).

Estas técnicas permitieron que Silvina se estabilizara y estabilizara su relación, de modo tal que a partir de ese momento fueron cada vez menos las veces en las que sucumbía al desborde.

Pasamos a explorar entonces otro aspecto de la situación, esto es, el contenido de sus atribuciones, según las cuales él no quería verla o estar con ella.

En este caso el trabajo fue de índole exploratoria, tratando de identificar las creencias disfuncionales que se hallaban en la base de ese contenido inicial (Grande, 2007). De este modo surgieron las siguientes creencias: "No me quiere ver a mí, quiere ver a otra que le interesa. No me quiere ver porque no le intereso. Está conmigo solamente para tener relaciones sexuales. No le intereso porque no estoy a su altura: él es perfecto y yo soy un desastre".

Como podemos ver, los dos temas principales eran la autodesvalorización y los celos. En la raíz de estos últimos se hallaba también una merma en el sentimiento de estima de sí, por lo cual los temas de "autoestima cero" y "no me quiero nada" adquirieron el mayor relieve en este punto (la sexualidad jugaba un papel de la siguiente forma: como ella se sabía sexualmente atractiva - "físicamente muy bien" —, suponía que era la única manera de lograr que él quisiera estar con ella. En un segundo momento, esta misma "solución" se le volvía en contra: él "sólo" quería "eso" de ella). 
La continuación del trabajo tomó ahora el rumbo de determinar con mayor claridad y detalle la representación de sí y la representación del objeto (desastre-perfecto), así como las resultantes de su interacción (ubicar a su pareja en el lugar del mentor, del que todo lo sabe, del que es social y culturalmente superior, mientras ella se ubicaba en el lugar complementario. De aquí surgía un intento de identificarse, tanto con él como con el tipo de mujer que ella suponía que él valoraba).

La contrapartida de esta idealización del otro aparecía en los momentos de crisis, en donde este quedaba caracterizado como un objeto totalmente "malo" (egoísta, mentiroso, traidor...y una serie de atribuciones insultantes), respecto del cual surgía un intenso afán de venganza (herirlo, degradarlo, hacerle sufrir lo que ella sufría, etc.).

Junto a esta indagación, fue posible reconstruir con cierto detalle una relación anterior, que había tenido a los 18 años, cuya similitud con la actual llegaba hasta los menores detalles. Esta concordancia permitió que Silvina pudiera identificar un patrón relacional que iba más allá de la persona de su pareja actual y que derivaba de un modo suyo de configurar los vínculos (Rudolf, 2013). La claridad conseguida acerca de este punto le permitió tomar mayor distancia de sus reacciones y de las supuestas "maldad" y "perfección" de su novio.

Asimismo, en la medida en que íbamos adquiriendo mayor comprensión sobre estos aspectos, llevamos a cabo una reconstrucción de su infancia, buscando llegar a las raíces de estas representaciones y de este patrón vincular.

En el núcleo de su problemática infantil encontramos la vivencia de una madre desconectada, que prefería a la hermana menor de Silvina (según la percepción de esta), un padre ausente y una larga serie de situaciones de abandono que resulta imposible reseñar en este lugar. De igual forma, pudimos inferir la existencia de conflictos tempranos (el afán de venganza hacia la madre y la hermana, sofocado y transferido a la relación actual con su pareja).

En este territorio se entrelazaban las fallas estructurales, los patrones relacionales, los conflictos y los sistemas motivacionales, en un complejo y rico entramado, en el que se hacía necesario implementar también la utilización de otras técnicas, como la exploración, el señalamiento y la interpretación.

\section{Conclusión}

Deseo terminar este trabajo enfatizando la importancia de llevar a cabo una evaluación precisa del peso relativo que poseen el conflicto y/o el déficit estructural en el padecer del paciente que nos consulta.

Tal como he intentado mostrar, esta evaluación tiene profundas implicaciones para la elección del encuadre, de la actitud terapéutica, de la selección de focos de trabajo, del modo de trabajar con los patrones relacionales y del tipo de intervenciones que se privilegian.

Considero que el estudio detenido de estos criterios y su aplicación sistemática pueden enriquecer, de modo considerable, el acerbo con que cuenta el terapeuta, para llevar a cabo su tarea en la práctica clínica.

\section{Referencias}

Allen, J. G., Fonagy, P. y Bateman, A. (2008). Mentalizing in Clinical Practice. Arlington, EstadosUnidos: American Psychiatric Publishing.

Bateman, A., Fonagy, P. (2006). Mentalization-Based Treatment for Borderline Personality Disorder. A practical Guide. Oxford, Reino Unido: Oxford University Press.

Bruch, H. (1973). Les Yeuxet le Ventre, l'obèse, l'anorexique. París, Francia: Editions Payot.

Cierpka, M. (2004). Geleitwort. En G. Rudolf, Strukturbezogene Psychotherapie. Leitfaden zur psychodynamischen Therapiestruktureller Störungen (pp. VII-X). Stuttgart, Alemania: Schattauer.

Dagnino Robles, P. A. (2012). Focus in Psychotherapy: Characteristics and trajectories through the therapeutic process (Tesis doctoral). Universidad de Heidelberg, Alemania. Recuperado de https://www.researchgate. net/publication/281657017 Focus in Psychotherapy Characteristics and trajectories through the therapeutic process 
Dreher, C. (2006). Persönlichkeitsstrukturelle und symptombezogene Veränderungen während der psychoanalytisch orientierten Psychotherapie im stationären Behandlungssetting. Eine naturalistische Verlaufuntersuchung in der Littenheid Klinik für Psychiatrie und Psychotherapie. (Tesis doctoral). Universidad de Basel, Alemania. Recuperado de https://edoc.unibas.ch/782/

Fonagy, P., Gergely, G., Jurist, E. y Target, M. (2002). Affect regulation, mentalization and the development of the self. Nueva York, Estados Unidos: Other Press.

Freud, S. (1980a). Análisis de la fobia de un niño de cinco años. En Obras Completas (Tomo X, pp. 7-118). Buenos Aires, Argentina: Amorrortu Editores. (Obra original publicada en 1909).

Freud, S. (1980b). Sobre los tipos de contracción de neurosis. En Obras Completas (Tomo XII, pp. 239-245). Buenos Aires, Argentina: Amorrortu Editores. (Obra original publicada en 1912).

Freud, S. (1979). Algunos tipos de carácter dilucidados por el trabajo analítico: II Los que fracasan cuando triunfan. En Obras Completas (Tomo XIV, pp. 323-337). Buenos Aires, Argentina: Amorrortu Editores. (Obra original publicada en 1916).

Fürstenau, P. (1977). Die beiden Dimensionen des psychoanalytischen Umgangs mit strukturell Ich-gestörten Patienten. Ein Beitragzur Erweiterung der psychoanalytischen Praxeologie. Psyche. ZeitschriftfüPsychoanalyse, 31(3), 197-207.

Grande, T. (2002). Therapeutische Haltungen im Umgang mit "Struktur" und "Konflikt". En G. Rudolf, T. Grandey y P. Henningsen, Die Struktur der Persönlichkeit. Teoretische Grundlagen zur Psychodynamischen Therapie Struktureller Störungen (pp. 236-248). Alemania, Stuttgart: Schattauer.

Grande, T. (2007). Wie stellen sich Konflikt und Struktur in Beziehungen dar? Zeitschriftfür Psychosomatische Medizin und Psychotherapie, 53(2), 144-162.

Greenson, T. (1976). Technique and practice of Psychoanalysis. Madison, Estados Unidos: International Universities Press.

Grupo de trabajo OPD-2. (2008). Diagnóstico Psicodinámico Operacionalizado. Manual para el diagnóstico, indicación y planificación de la psicoterapia. Barcelona, España: Herder Editorial.

Helman, N. (1992). Un tipo de trastorno de la personalidad: el paciente con self incompleto. Psicoanálisis, 14(2), 269-290.

Horowitz, M., Kernberg, O. y Weinshel, E. (1993). Psychic Structure and Psychic Change. Essays in Honor of Robert S. Wallerstein, M. D. Madison, Estados Unidos: International Universities Press.

Juen, F. (2005). Das Denken über das Denken und Fühlen. Marburgo, Alemania: Tectum Verlag.

Kernberg, O. (1979). Desórdenes fronterizos y narcisismo patológico. Buenos Aires, Argentina: Paidós. (Obra original publicada en 1975).

Killingmo, H. (1989). Conflicto y déficit: implicaciones para la técnica. Libro Anual dePsicoanálisis, 15,112-126. Kohut, H. (1989). Análisis del self. El tratamiento psicoanalítico de los trastornos narcisistas de la personalidad. Buenos Aires, Argentina: Amorrortu Editores. (Obra original publicada en 1971).

Lanza Castelli, G. (2016a).Comparación entre la histeria y los pacientes fronterizos desde la perspectiva de André Green, ilustrada con el análisis de un caso clínico. Clínica Contemporánea, 7, 167-179. https://doi. org/10.5093/cc2016a14

Lanza Castelli, G. (2016b). Conflicto, estructura y mentalización. Interrelaciones y complementariedades. Aperturas Psicoanalíticas, (50). Recuperado de https://aperturas.org/articulo.php?articulo=908\&a=Conflicto-estructura-y-mentalizacion-interrelaciones-y-complementariedades

Lecours, S. (2007). Supportive interventions and non symbolic mental functioning. International Journal of Psychoanalysis, 88, 895-915.

Lieberman, M. D. Eisenberger, N. I. y Crockett, M. J. (2007). Putting Feelings into words: affect labeling disrupts amygdale activity to affective stimuli. Psychological Science, 18, 421-428.

Mentzos, S. (2017). Neurotische Konfliktverarbeitung. Enführung in die psychoanalytische Neurosenlehre unter Berücksichtigung neuer Perspekiven. Frankfurt am Main, Alemania: Fischer. (Obra original publicada en 1982). Pouget-Schors, D. (2002). Strukturkonzepte in der psychoanalytischen Entwicklung. En G. Rudolf, T. Grande y P. Henningsen, DieStruktur der Persönlichkeit. Teoretische Grundlagen zur Psychodynamischen Therapie Struktureller Störungen (pp. 49-67). Stuttgart, Alemania: Schattauer. 
Rapaport, D. (1967). La estructura de la teoría psicoanalítica, Buenos Aires, Argentina: Paidós. (Obra original publicada en 1960).

Resch, F. (2002). Strukturunde Strukturveränderung im Kindes- und Jugendalter. En G. Rudolf, T. Grande, y P.Henningsen, Die Struktur der Persönlichkeit. Teoretische Grundlagen zur Psychodynamischen Therapie Struktureller Störungen (pp. 116-131). Stuttgart, Alemania: Schattauer.

Rudolf, G. (2002a). Struktur als psychodynamisches Konzept der Persönlichkeit. En G. Rudolf, T. Grande, y P.Henningsen, Die Struktur der Persönlichkeit. Teoretische Grundlagen zur Psychodynamischen Therapie Struktureller Störungen (pp. 2-48). Stuttgart, Alemania: Schattauer.

Rudolf, G. (2002b). Strukturbezogene Psychotherapie. En G. Rudolf, T. Grande, y P. Henningsen, Die Struktur der Persönlichkeit. Teoretische Grundlagen zur Psychodynamischen Therapie Struktureller Störungen (pp. 249-271). Stuttgart, Alemania: Schattauer.

Rudolf, G. (2004). Leitfaden zur psychodynamischen Therapie struktureller Störungen. Stuttgart, Alemania: Schattauer.

Rudolf, G. (2007). Strukturbezogene Psychotherapie der Borderline- Persönlichkeitsstörung. En G. Dammann y P. L. Janssen, Psychotherapie der Borderline-Störungen. Krankheitsmodelle und Therapiepraxisstörungsspezifisch und schulenübergreifend (pp. 89-98). Stuttgart, Alemania: Georg ThiemeVerlag KG.

Rudolf, G. (2010) Psychodynamische Psychotherapie. Die Arbeit an Konflikt, Struktur und Trauma. Alemania, Stuttgart: Schattauer.

Rudolf, G. (2013). Strukturbezogene Psychotherapie. Leitfaden zur psychodynamischen Therapie struktureller Störungen. Stuttgart, Alemania: Schattauer.

Rudolf, G. y Grande, T. (2006). Fokusbezogene psychodynamische Psychotherapie. Ein OPD basierte Leinfaden. Psychotherapeut, 51, 276-289.

Schüßler, G. (2004). Innerpsychischerkonflikt und struktur: Wo steht das unbewusste heute? En R. W. Dahlbender, P. Buchheim y G. Schüßler (Eds.), Lernen an der Praxis. OPD und die qualitätssicherung in der psychodynamischen Psychotherapie (pp. 181-192). Berna, Alemania: Huber.

Schüßler, G., Burgmer, M., Mans, E., Schneider, G., Dahlbender, R. y Heuft, G. (2006). Neurotische Konfliktsystematik - Grundlagen der Konfliktdiagnostik (OPD-2). Zeitschrift für Psychosomatische Medizin und Psychotherapie 52, 23-38.

Smith, H. F. (2003). Conception of conflict in psychoanalytic theory and practice. Psychoanalytic Quarterly, 72, 49-96.

Stass, M., Grande, T., Janssen, P., Oberbracht, C. y Rudolf, G. (2014). OPD-2 im Psychotherapie-Antrag. Psychodynamische Diagnostik und Fallformulierung. Verlag, Alemania: Hans Huber.

Sugarman, A. (2006). Mentalization, insightfulness, and therapeutic action. The importance of mental organization. International Journal of Psychoanalysis, 87, 965-87.

Artículo recibido: 09/12/2018

Artículo aceptado: 09/05/2019 